\title{
Measurement of hard double-parton interactions with the ATLAS detector
}

\section{Timothy A. D. Martin*}

On behalf of the ATLAS collaboration

University of Warwick

E-mail: tim.martinecern.ch

\begin{abstract}
The production of a $W$ boson in association with two jets was studied in proton-proton collisions at a centre-of-mass energy of $\sqrt{s}=7 \mathrm{TeV}$ with the ATLAS detector at the LHC. Data corresponding to an integrated luminosity of $36 \mathrm{pb}^{-1}$ were analysed for the presence of doubleparton interactions. The fraction of events arising from double-parton interactions was extracted using the momentum balance of the two jets, and was evaluated to be $f_{\mathrm{DP}}^{\text {Detector }}=$ $0.08 \pm 0.01$ (stat.) \pm 0.02 (syst.) for jets with transverse momentum $p_{\mathrm{T}}>20 \mathrm{GeV}$ and rapidity $|y|<2$. This is expressed as a measure of the effective area parameter for hard double-parton interactions, $\sigma_{\text {eff }}=15 \pm 3$ (stat. $)_{-3}^{+5}$ (syst.) mb.
\end{abstract}

International Conference on the Structure and the Interactions of the Photon including the 20th International Workshop on Photon-Photon Collisions and the International Workshop on High Energy Photon Linear Colliders

20 - 24 May 2013

Paris, France

* Speaker. 


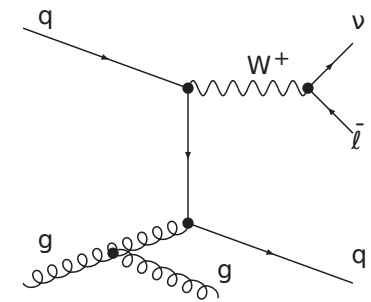

(a)

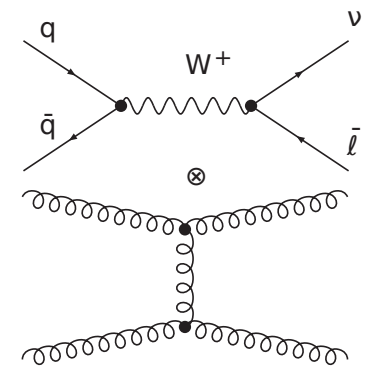

(b)

Figure 1: Example leading order Feynman diagrams for (left) the direct production of two jets in association with a $W$ boson and (right) the double-parton interaction production of the same final state.

\section{Introduction}

The presence of multiple hard scale interactions in hadron-initiated processes has been discussed since the early days of the parton model $[1,2,3]$. Perturbative quantum chromodynamical (QCD) formulations have been calculated for many experimental signatures including the production of four jets [4], double Drell-Yan [5] and the production of a $W$ boson in association with two jets; the process studied in this analysis [6] by the ATLAS collaboration at the Large Hadron Collider (LHC).

The effect of double-parton interactions (DPI) is parametrised by an effective cross section $\sigma_{\text {eff }}(s)$ which quantifies the probability of a second hard scatter, given that one has occurred. In this sense $\sigma_{\text {eff }}(s)$ is related to the flux of accompanying partons in the proton which is inversely proportional to the proton's cross sectional area. The effective cross section is defined as

$$
\sigma_{\mathrm{eff}}(s)=\frac{\hat{\sigma}_{W}(s) \cdot \hat{\sigma}_{2 \mathrm{j}}(s)}{\hat{\sigma}_{W+2 \mathrm{j}}^{(\mathrm{tot})}(s)-\hat{\sigma}_{W+2 \mathrm{j}}^{(\mathrm{SPI})}(s)}
$$

where $\hat{\sigma}_{W}(s)$ and $\hat{\sigma}_{2 \mathrm{j}}(s)$ are respectively the differential cross sections for the production of a $W$ boson and di-jet system while $\hat{\sigma}_{W+2 \mathrm{j}}(s)$ is the differential cross section of a $W$ boson produced along with exactly two jets. Here (tot) specifies all events while (SPI) is the 'single parton interaction' subset of events for which the two jets are produced in direct association with the $W$ boson.

This difference is shown in Figure 1 where the leading order diagrams for the SPI and DPI production mechanisms are presented. The kinematics of the two different production methods alter the correlations of the final state in an observable way. Most notably, the QCD radiation responsible for the second jet in the SPI process results in a di-jet system where the two jets have a tendency to be emitted co-linear to each other, while for the DPI process the two interactions arec considered independent and the resulting di-jet system should balance back-to-back in the transverse plane.

The amount of DPI is an important quantity to study for MC tuning purposes and to constrain background estimations in other sensitive channels such as $W+b$-jets [7] in which the DPI correction was non-negligible for low $p_{\mathrm{T}} b$-jets. 


\section{Analysis Strategy}

In order to calculate $\sigma_{\text {eff }}$, it is first required to measure the fraction of DPI in events which contain a single $W$ boson (decaying to either $e v_{e}$ or $\mu v_{\mu}$ ) along with exactly two jets. This fraction, calculated with detector-level reconstructed objects is defined as

$$
f_{\mathrm{DP}}^{\text {Detector }}=\frac{N_{W_{0 \mathrm{j}}+2 \mathrm{j}_{\mathrm{DPI}}}}{N_{W_{2 \mathrm{j}}}+N_{W_{0 \mathrm{j}}+2 \mathrm{j}_{\mathrm{DPI}}}}
$$

where $N_{W_{2 j}}$ is the number of events with the jets directly associated with the production of the $W$ and $N_{W_{0 j}+2 j_{D P I}}$ is the number of events where the jets originate from DPI.

\subsection{ATLAS Data Selection}

The ATLAS 2010 dataset is used, corresponding to $36 \mathrm{pb}^{-1}$ of integrated luminosity collected while all necessary subsystems were operational. The ATLAS detector is described in [8]. Electron candidates were reconstructed with transverse momentum ${ }^{1} p_{\mathrm{T}}>20 \mathrm{GeV}$ and pseudorapidity $|\eta|<$ 2.47 , muon candidates were reconstructed with $p_{\mathrm{T}}>20 \mathrm{GeV}$ and $|\eta|<2.4$ and jet candidates with $p_{\mathrm{T}}>20 \mathrm{GeV}$ and rapidity $|y|<2.8$. Events were additionally required to satisfy $E_{\mathrm{T}}^{\text {miss }}>25 \mathrm{GeV}$ and $m_{\mathrm{T}}>40 \mathrm{GeV}$ where $m_{\mathrm{T}}$ is the reconstructed transverse mass ${ }^{2}$ of the $W$. This selection shares a great degree of similarity with [9]. Three datasets are defined using the above fiducial and kinematic selection which are used in the extraction of $f_{\mathrm{DP}}^{\text {Detector }}$ and then $\sigma_{\text {eff. }}$. They are a $W$ plus exactly zero jets, a $W$ plus exactly two jets and exactly two jets, independant of $W$ requirements. This latter di-jet sample is selected with a fully-unbiased and fully-efficient trigger based on the Minimum Bias Trigger Scintillators and Zero Degree Calorimeter.

\subsection{Monte Carlo and Backgrounds}

Two Monte Carlo simulations were used to quantify corrections and assess the analysis strategy in [6]. The primary signal MC was a $W$ boson sample generated with ALPGEN interfaced with HERWIG and JIMMY (AUET2 tune) using the MLM matching scheme, hereafter A+H+J. Additionally SHERPA was used with CKKW merging and the default underlying event tune. Both generators used the CTEQ6L1 parton distribution functions.

Background contributions normalised to theoretical cross sections were simulated with POWHEG $(t \bar{t})$, MC@ NLO (single top, di-boson) and Pythia6 $(Z \rightarrow l l)$. The multi-jet background was assessed in a data-driven manner by inverting certain calorimeter shower shape $(e)$ or impact parameter $(\mu)$ requirements to create a multi-jet enhanced sample which was normalised to the shape of the $E_{\mathrm{T}}^{\text {miss }}$ distribution. Backgrounds amount to $19 \%$ of the electron channel (dominantly multi-jet and $W \rightarrow \tau v_{\tau}$ ) and $14 \%$ of the muon channel (dominantly multi-jet and $Z \rightarrow l l$ ).

\footnotetext{
${ }^{1}$ ATLAS uses a right-handed coordinate system with its origin at the nominal interaction point (IP) in the centre of the detector and the $z$-axis along the beam pipe. The $x$-axis points from the IP to the centre of the LHC ring, and the $y$-axis points upward. Cylindrical coordinates $(r, \phi)$ are used in the transverse plane, $\phi$ being the azimuthal angle around the beam pipe. The pseudorapidity is defined in terms of the polar angle $\theta$ as $\eta=-\ln \tan (\theta / 2)$.

${ }^{2}$ Calculated as $m_{\mathrm{T}}=\left[2 p_{\mathrm{T}}^{l} E_{\mathrm{T}}^{\text {miss }}\left(1-\cos \Delta \phi_{l, E_{\mathrm{T}}^{\text {miss }}}\right)\right]$ with $\Delta \phi_{l, E_{\mathrm{T}}^{\text {miss }}}$ being the interval in $\phi$ between the lepton $p_{\mathrm{T}}$ vector and the $E_{\mathrm{T}}^{\mathrm{miss}}$ vector.
} 


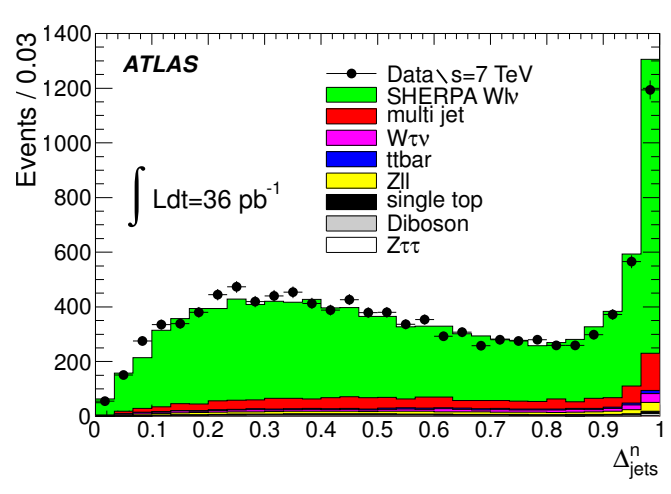

(a)

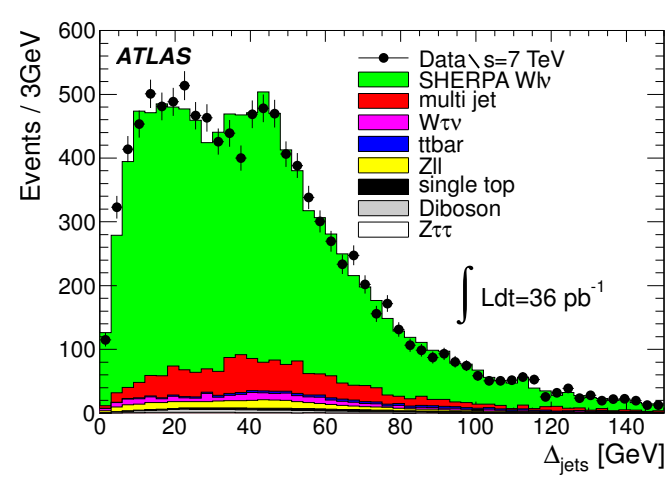

(b)

Figure 2: Distribution of data compared to SHERPA MC and backgrounds of the two discriminating variables used in the analysis, $\Delta_{\text {jets }}^{\mathrm{n}}$ and $\Delta_{\text {jets }}$.

\subsection{Analysis Variables and Fit Templates}

The fraction of $W$ plus two jet events where the jets arise from multiple parton interaction $\left(f_{\mathrm{DP}}^{\text {Detector }}\right)$ is extracted using a fit to data of a linear sum of two templates. The variables fitted are designed to distinguish co-linear di-jet systems from those which balance in the transverse plain. The variables

$$
\Delta_{\text {jets }}=\left|\vec{p}_{\mathrm{T}}^{\mathrm{J1}}+\vec{p}_{\mathrm{T}}^{\mathrm{J} 2}\right| \quad \text { and } \quad \Delta_{\text {jets }}^{\mathrm{n}}=\frac{\left|\vec{p}_{\mathrm{T}}^{\mathrm{J} 1}+\vec{p}_{\mathrm{T}}^{\mathrm{J} 2}\right|}{\left|\vec{p}_{\mathrm{T}}^{\mathrm{J}}\right|+\left|\vec{p}_{\mathrm{T}}^{\mathrm{J}}\right|}
$$

are used where $\mathrm{J} 1$ and $\mathrm{J} 2$ label the two jets. The absolute transverse momentum normalised di-jet balance $\Delta_{\text {jets }}^{\mathrm{n}}$ is the preferred discriminator as it is less sensitive to systematic effects from the jet energy scale uncertainty than $\Delta_{\text {jets }}$. The data are compared to MC, in this case SHERPA, along with evaluated backgrounds in Figure 2. A good agreement is observed. The first of the two templates used to extract $f_{\mathrm{DP}}^{\text {Detector }}$ from these distributions is referred to as Template A and contains only events in which the di-jet system is produced in direct association with the $W$. It is modelled using the MC and hence requires the removal of events in MC where the di-jet system arose from additional MPI. In $\mathrm{A}+\mathrm{H}+\mathrm{J}$, these hard MPI are removed by vetoing events which contain two or more secondary partons above a cut-off scale, $p_{\mathrm{T}}^{\max }=15 \mathrm{GeV}$ (the choice of this cut is explored in Section 2.4). For SHERPA, the only available option is less desirable: switching off MPI altogether, resulting in effectively no secondary pertubative scatters in the range $p_{\mathrm{T}}>3.5 \mathrm{GeV}$. The effect of vetoing hard MPI events is illustrated in Figure 3, events are predominately vetoed at low $\Delta_{\text {jets }}^{\mathrm{n}}$, corresponding to di-jet systems which balance in $p_{\mathrm{T}}$.

Template B is used to model di-jet systems where the di-jet arises from MPI. The data sample of exactly two jets is used for this purpose.

\subsection{The Effect of $p_{\mathrm{T}}^{\max }$}

The generator cut on $p_{\mathrm{T}}^{\max }$ should remove hard MPI scatters which produce di-jets with $p_{\mathrm{T}}>$ $20 \mathrm{GeV}$ yet retain soft MPI and the rest of the underlying event so as to better match the soft dynamics present in data. In $\mathrm{A}+\mathrm{H}+\mathrm{J}$ the cut was studied over the range $3.5<p_{\mathrm{T}}^{\max }<20 \mathrm{GeV}$. It 


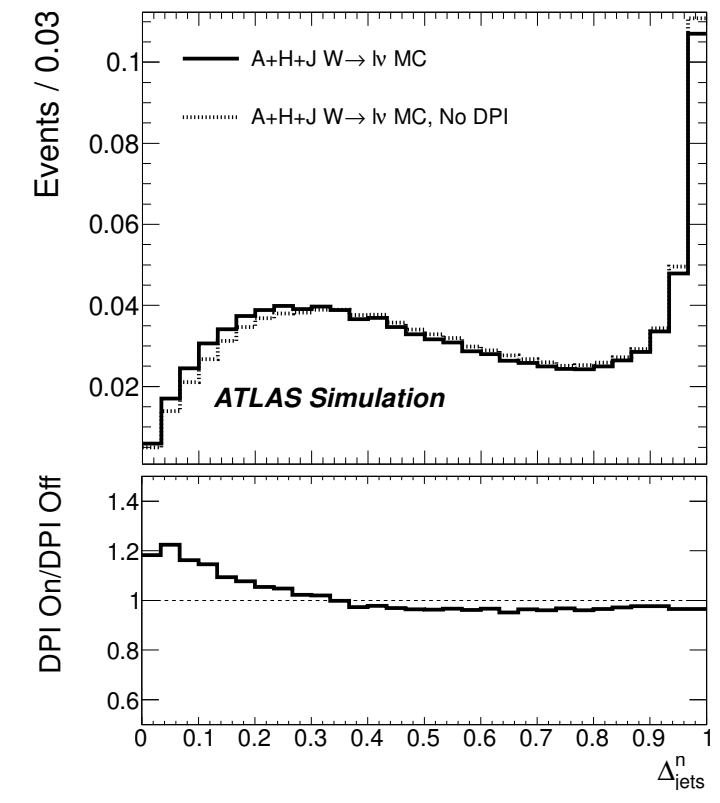

(a)

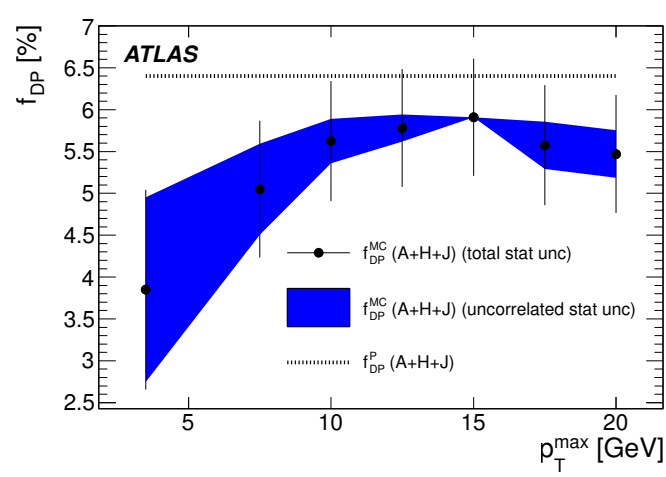

(b)

Figure 3: (a) Inclusive $\mathrm{A}+\mathrm{H}+\mathrm{J}$ MC compared to $\mathrm{MC}$ with hard MPI vetoed (Template A). (b) Effect of variation of the cut $p_{\mathrm{T}}^{\max }$ determining the hard scale for MPI in $\mathrm{A}+\mathrm{H}+\mathrm{J}$ compared to the fraction of MPI in the MC (dotted line). The shaded band denotes the fraction of the statistical error which is correlated with respect to the nominal cut at $p_{\mathrm{T}}^{\max }=15 \mathrm{GeV}$.

was observed that setting the cut at $3.5 \mathrm{GeV}$ to approximately match SHERPA resulted in agreement between the two MCs to within $10 \%$. The optimal cut at $p_{\mathrm{T}}^{\max }=15 \mathrm{GeV}$ gives the best agreement with the parton level fraction of MPI in the MC. The agreement is within 10\% and is interpreted as being equivalent to a measurement at the parton level to within this accuracy.

\section{Determination of $\mathbf{f}_{\mathrm{DP}}^{\text {Detector }}$}

The backgrounds discussed in Section 2.2 are subtracted from the data and the data are fitted with a linear combination of Template A and Template $\mathrm{B}$. The two highest bins of $\Delta_{\text {jets }}^{\mathrm{n}}$ are excluded as they correspond to parallel jets and therefore rather test the parton shower model. The data fitted with the $\mathrm{A}+\mathrm{H}+\mathrm{J}$ no-MPI Template $\mathrm{A}$ and data di-jet template $\mathrm{B}$ are shown in Figure 4. A correction is applied to $f_{\mathrm{DP}}^{\text {Detector }}$ to account for the effects in data of multiple interactions per bunch crossing ${ }^{3}$ (pile-up). The correction factor is $r_{\text {pile-up }}=1.17 \pm 0.15$ (stat.). The fraction of MPI is determined using the $\Delta_{\text {jets }}^{\mathrm{n}}$ discriminator to be

$$
\left.f_{\mathrm{DP}}^{\text {Detector }}\left(\Delta_{\text {jets }}^{\mathrm{n}}\right)=0.076 \pm 0.013 \text { (stat. }\right) \pm 0.018 \text { (syst.). }
$$

The fit quality was $\chi^{2} / N_{\mathrm{DOF}}=37 / 28$ and the breakdown of uncertainties on $f_{\mathrm{DP}}^{\text {Detector }}$ is listed in Table 1.

\footnotetext{
${ }^{3}$ There were insufficient statistics in the $W$ sample to perform the analysis in low pile-up data. However the effect of pile-up was quantified using early di-jet data and $\mathrm{A}+\mathrm{H}+\mathrm{J} \mathrm{MC}$ with no pile-up.
} 


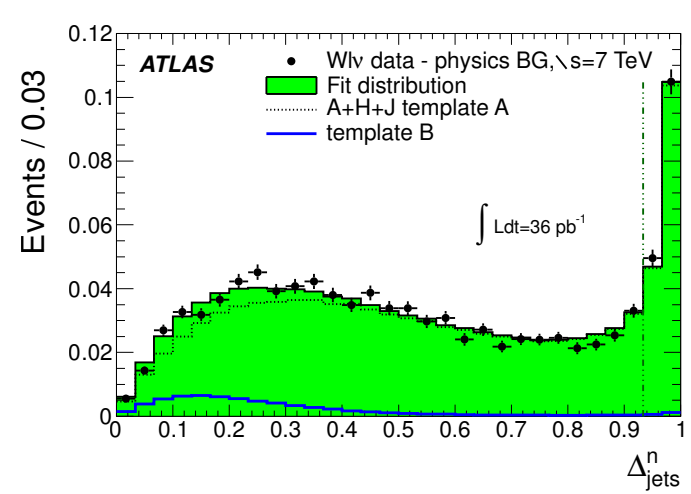

(a)

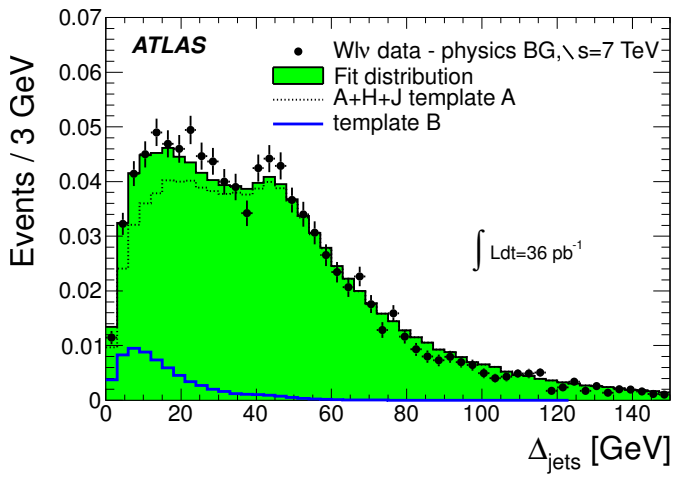

(b)

Figure 4: Distribution of $\Delta_{\text {jets }}^{\mathrm{n}}$ and $\Delta_{\text {jets }}$ with background-subtracted data and the best fit values of Template A and Template B. The two highest bins of $\Delta_{\text {jets }}^{\mathrm{n}}$ are excluded from the fit.

Table 1: Table of sources of uncertainty on $f_{\mathrm{DP}}^{\text {Detector }}$. The theory uncertainty includes both the effect of using SHERPA and the uncertainty from the choice of $p_{\mathrm{T}}^{\text {cut }}$.

\begin{tabular}{l|r} 
Source & Uncertainty \\
\hline Theory & $10 \%$ \\
Pile-up & $13 \%$ \\
Jet Energy Scale & $12 \%$ \\
Jet Energy Resolution & $8 \%$ \\
Background Modelling \& Lepton Response & $11 \%$ \\
\hline Total Systematic & $\mathbf{2 4 \%}$ \\
Total Statistical & $\mathbf{1 7 \%}$
\end{tabular}

\section{Hadron-level Studies}

The key distributions used to determine $f_{\mathrm{DP}}^{\text {Detector }}$ were unfolded to correct the data for detector effects. The RooUnfold package [10] was used to correct the background-subtracted data using two iterations of Bayesian unfolding [11]. The hadron level MC used in the correction utilised the same cuts as applied on the detector level, applied to hadron level quantities and jets formed from hadron-level particles. This data are of use for MC tuning purposes or for alternate approaches to extracting MPI related quantities.

\section{Determination of $\sigma_{\text {eff }}$}

When converted from cross sections to event yields $(N)$, equation (1.1) becomes

$$
\sigma_{\mathrm{eff}}=\left(\frac{N}{\mathscr{A} C \cdot \varepsilon \mathscr{L}}\right)_{W+0 \mathrm{j}} \cdot\left(\frac{N}{\mathscr{A} C \cdot \varepsilon \mathscr{L}}\right)_{2 \mathrm{j}} \cdot\left(\frac{\mathscr{A} C \cdot \varepsilon \mathscr{L}}{f_{\mathrm{DP}}^{\text {Detector }} \cdot N}\right)_{W+2 \mathrm{j}}^{\mathrm{DPI}} .
$$

As the $W+0 \mathrm{j}$ and $W+2 \mathrm{j}^{\mathrm{DPI}}$ samples are selected with the same trigger, luminosity $\mathscr{L}$ and trigger efficiency $\varepsilon$ cancel. In addition, through the assumption that MPI are not correlated to the primary 


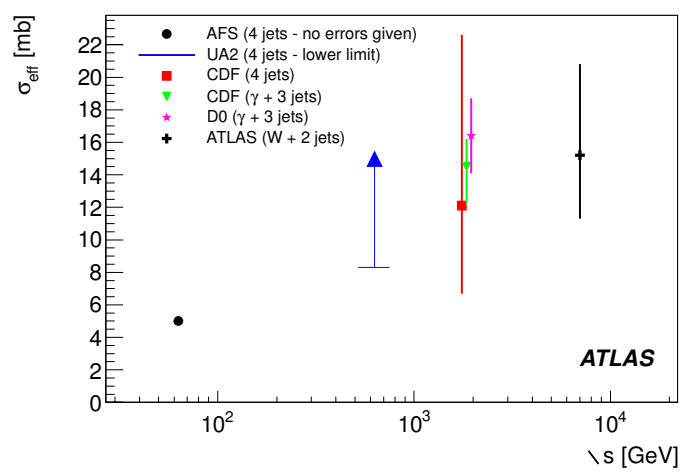

Figure 5: Dependence of $\sigma_{\text {eff }}$ as a function of centre-of-mass energy for different experiments $[12,13,14,15,16]$. Offsets applied to $1.8 \mathrm{TeV}$ data for clarity.

scatter, the acceptance and correction factors $\mathscr{A}$ and $C$ cancel. This leaves the efficiency of the di-jet sample, which was fully efficient $\left(\varepsilon_{2 j}=1\right)$, plus an additional uncertainty on the luminosity of the di-jet sample (3\%). The dominant uncertainty on $\sigma_{\text {eff }}$ is the propagation of the uncertainty on $f_{\mathrm{DP}}^{\text {Detector }}(24 \%)$. An addition uncertainty on the response to leptons and background in evaluating the exclusivity ration $N_{W+0 \mathrm{j}} / N_{W+2 \mathrm{j}}^{\mathrm{DPI}}(5 \%)$ is also included. The effective cross section is evaluated as

$$
\left.\sigma_{\text {eff }}(\sqrt{s}=7 \mathrm{TeV})=15 \pm 3 \text { (stat. }\right)_{-3}^{+5} \text { (syst.)mb. }
$$

This value is consistent with values measured previously in other experiments at lower centre of mass energies and in different channels as shown in Figure 5.

\section{Conclusion}

The double-parton interaction fraction $f_{\mathrm{DP}}^{\text {Detector }}$ for events containing a $W$ boson and exactly two jets has been extracted from $p p$ collisions in the ATLAS detector at $\sqrt{s}=7 \mathrm{TeV}$ using $36 \mathrm{pb}^{-1}$ of integrated luminosity. A central value of $f_{\mathrm{DP}}^{\text {Detector }}\left(\Delta_{\text {jets }}^{\mathrm{n}}\right)=0.076 \pm 0.013$ (stat.) \pm 0.018 (syst.) is obtained for jets with $p_{\mathrm{T}}>20 \mathrm{GeV}$ and $|y|<2.8$.

There is good agreement between MC and data in terms of the measured rate and kinematics of MPI. The measured fraction is used to calculate the effective parameter, $\sigma_{\text {eff }}$, which is consistent with previous measurements at lower centre-of-mass energies.

\section{References}

[1] P. Landshoff, J. Polkinghorne, Phys.Rev. D18, 3344 (1978)

[2] F. Takagi, Phys.Rev.Lett. 43, 1296 (1979)

[3] C. Goebel, F. Halzen, D. Scott, Phys.Rev. D22, 2789 (1980)

[4] B. Humpert, R. Odorico, Phys.Lett. B154, 211 (1985)

[5] M. Mekhfi, Phys.Rev. D32, 2371 (1985)

[6] ATLAS Collaboration, New Journal of Physics 15(3), 033038 (2013) 
[7] ATLAS Collaboration, JHEP 1306, 084 (2013)

[8] ATLAS Collaboration, JINST 3(08), S08003 (2008)

[9] ATLAS Collaboration, Phys.Rev. D85, 092002 (2012)

[10] T. Adye, arXiv:1105.1160 (2011)

[11] G. D’Agostini, arXiv:1010.0632 (2010)

[12] Axial Field Spectrometer Collaboration, Z.Phys. C34, 163 (1987)

[13] UA2 Collaboration, Phys.Lett. B268, 145 (1991)

[14] CDF Collaboration, Phys.Rev. D47, 4857 (1993)

[15] CDF Collaboration, Phys.Rev. D56, 3811 (1997)

[16] D0 Collaboration, Phys.Rev. D81, 052012 (2010) 